\title{
Perceived sucrose intensity is related to 6-n-propylthiouracil sensitivity and to markers of sugar intake
}

\author{
E. Feeney, S. O’Brien, A. Scannel, A. Markey and E. R. Gibney \\ UCD Centre for Food \& Health, School of Agriculture, Food Science \& Veterinary Medicine, University College Dublin, \\ Belfield, Dublin 4, Republic of Ireland
}

Taste perception of 6-n-propylthiouracil (PROP) can vary in individuals depending partly on polymorphisms of the bitter-taste receptor gene TAS $2 R 38^{(1)}$. Super-taster (ST) individuals are highly sensitive to the bitter taste of PROP, while non-tasters are much less sensitive and medium tasters lie between the two. Previous studies have shown that ST are highly sensitive not only to PROP, but also to the tastes of quinine and sucrose ${ }^{(2)}$ and can perceive differences in foods with altered fat content, possibly as a result of an increased number of trigeminal nerve fibres on the tongue ${ }^{(3)}$.The main aim of the present study was to examine some of the wide-ranging reasons for food choice within a group of children $(n$ 199) and their parents $(n$ 52) and to look at the role of PROP taster status in sucrose perception and sugar intake. Children aged 8-12 years and their parents were recruited from schools across Dublin. To determine taster status, PROPimpregnated paper discs were rated on the general labelled magnitude scale (gLMS) as previously described ${ }^{(4)}$. Subjects were then given a series of drinks of varying sucrose concentration, which they also rated on the gLMS. Average daily sugar intakes were obtained through a $3 \mathrm{~d}$ diet history, which was then analysed using WISP ${ }^{\odot}$ (Tinuviel Software, Llanfechell, Anglesey, UK). Values for non-milk extrinsic sugars (NMES) were taken as a marker for sugar intake.

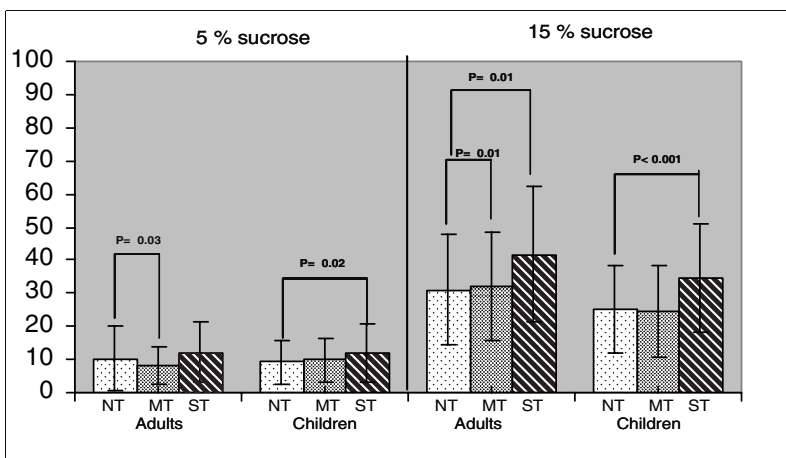

Fig. 1. Mean perceived sucrose intensity ratings for $5 \%$ and $15 \%(\mathrm{w} / \mathrm{v})$ sucrose in the three taster groups.

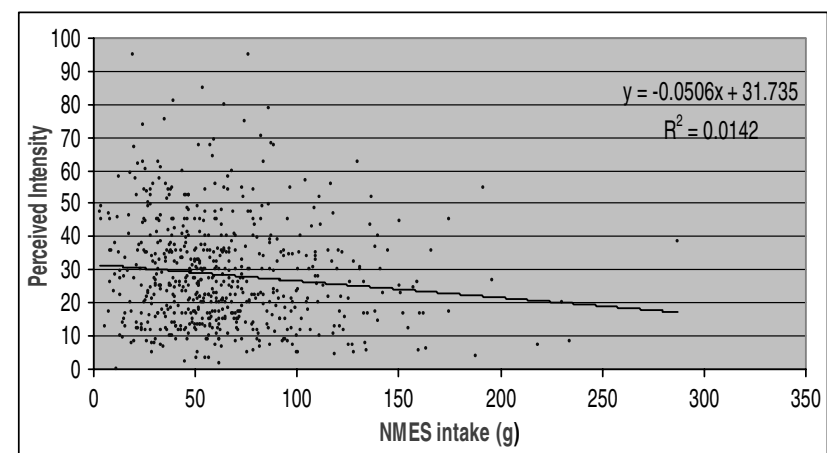

Fig. 2. Perceived intensity ratings $v$. NMES intakes (total population)

Non-tasters (NT) had significantly lower perceived sucrose intensity ratings in both adults and children, which was more pronounced for the higher sucrose solution. A slight negative correlation $(R-0.124, P<0.05)$ was observed in the total population between perceived sucrose intensity and mean daily intakes of NMES $(\mathrm{g})$. These results appear to support previous findings that PROP tasters perceive sucrose more intensely than NT and consequently consume less added sugar. The results presented here are part of a larger study aiming to examine the eating habits and reasons for food choice in Irish children and their parents.

The study is funded by the Food Institutional Research Measure (FIRM), Department of Agriculture and Food, Republic of Ireland.

1. Kim UK \& Drayna D (2004) Clin Genet 67, 275-280.

2. Delwiche JF (2001) Physiol Behav 74, 329-337.

3. Tepper BJ \& Nurse RJ (1997) Physiol Behav 61, 949-954.

4. Fast K, Green BG \& Bartoshuk L (1992) Appetite 39, 75. 